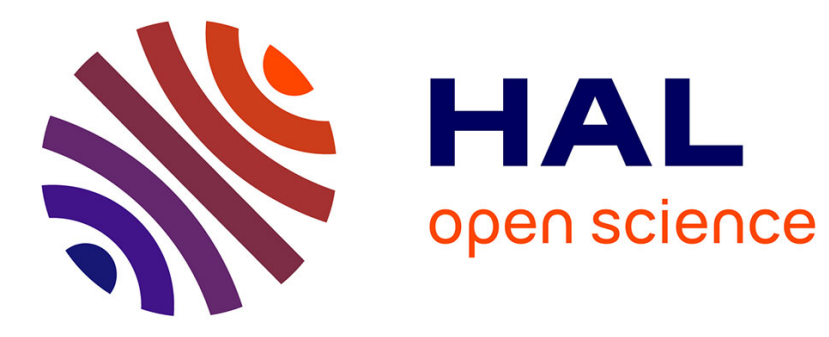

\title{
Low Complexity Adaptive Demapper for 2-D Non-Uniform Constellations
}

\author{
Jon Barrueco Gutierrez, Jon Montalban, Pablo Angueira, Charbel Abdel \\ Nour, Catherine Douillard
}

\section{- To cite this version: \\ Jon Barrueco Gutierrez, Jon Montalban, Pablo Angueira, Charbel Abdel Nour, Catherine Douillard. Low Complexity Adaptive Demapper for 2-D Non-Uniform Constellations. IEEE Transactions on Broadcasting, 2019, 65 (1), pp.10-19. 10.1109/TBC.2018.2811619 . hal-01745928}

\section{HAL Id: hal-01745928 \\ https://hal.science/hal-01745928}

Submitted on 28 Mar 2018

HAL is a multi-disciplinary open access archive for the deposit and dissemination of scientific research documents, whether they are published or not. The documents may come from teaching and research institutions in France or abroad, or from public or private research centers.
L'archive ouverte pluridisciplinaire HAL, est destinée au dépôt et à la diffusion de documents scientifiques de niveau recherche, publiés ou non, émanant des établissements d'enseignement et de recherche français ou étrangers, des laboratoires publics ou privés. 


\title{
Low Complexity Adaptive Demapper for Two- Dimensional Non-Uniform Constellations
}

\author{
J. Barrueco, Member, IEEE, J. Montalban, Member, IEEE, P. Angueira, Member, IEEE, \\ C. Abdel Nour, Member, IEEE and C. Douillard, Member, IEEE
}

\begin{abstract}
In this paper, a novel demapper for two-dimensional non-uniform constellations (2D-NUC) is proposed, exploiting the characteristics of these constellations. It represents the combination of two underlying demapping techniques targeting ATSC 3.0 compliant OFDM transceiver. On the one hand, for low code rates, we define a metric to perform condensed demapping. On the other hand, for high code rates, adaptive sub-region demapping is proposed. In this work, a combination of both demapping methods is designed showing comparable performance to the classical Max-log demapper since the gap does not exceed $0.1 \mathrm{~dB}$ for all code rates of the ATSC 3.0 standard. Higher complexity reduction, from $79.2 \%$ to $95.4 \%$, than state of art 2D-NUC demappers is obtained for 2D256NUCs. These results are validated for ideal and non-ideal channel state information (CSI) over additive white noise Gaussian (AWGN) and Rayleigh independently and identically distributed (iid) channels. Results are extended to 2D-1kNUCs and $2 \mathrm{D}-4 \mathrm{kNUCs}$ showing demapping complexity reduction from $96 \%$ to $99.7 \%$ with a negligible impact on performance.
\end{abstract}

Index Terms- ATSC 3.0, Broadcasting, demapping complexity, non-uniform constellations.

\section{INTRODUCTION}

I ncreasing spectral efficiency of wireless broadcasting systems to get close to the channel capacity upper bound represents nowadays a major goal of many research projects worldwide. Techniques such as Multiple Input Multiple Output (MIMO) [1], Rotated Constellations (RC) [2] and NonUniform Constellations (NUC) [3] respond to this demand providing a good tradeoff between system capacity and bandwidth usage.

A paradigmatic standardization example is the newest digital terrestrial broadcasting system called ATSC 3.0 [4].

This work was supported in part by the Basque Government (PREDOC Program and IT-683-13), in part by the University of the Basque Country (UFI 11/30) and in part by the Spanish Ministry of Economy and Competitiveness (project 5G-newBROS under Grant TEC2015-66153-P MINECO/FEDER, UE)

J. Barrueco, and P. Angueira are with the Department of Communications Engineering, University of the Basque Country, 48013 Bilbao, Spain (e-mail: jon.barrueco@ehu.eus; jon.montalban@ehu.eus; pablo.angueira@ehu.eus).

J. Montalban is with the Department of Electronic Technology, University of the Basque Country, 20018 Donostia, Spain (e-mail: jon.montalban@ehu.eus).

C. A. Nour and C. Douillard are with the Institut Mines-Télécom/Télécom Bretagne, CNRS Lab-STICC, UMR 3192, Brest 29238, France, and also with the Université Européenne de Bretagne, Brest, France (e-mail: firstname.lastname@imt-atlantique.fr).
This standard adopts MIMO as an optional technology [5] and it also includes NUCs [6] to provide performance gains up to $1.8 \mathrm{~dB}$ (compared to classical Quadrature Amplitude Modulations (QAM)). NUCs are designed to maximize the Bit Interleaved-Coded Modulation (BICM) channel capacity [7] [9].

There are two possible NUC families: 1-Dimensional NUC (1D-NUC) and 2-Dimensional NUC (2D-NUC). 1D-NUCs were already included in Digital Video Broadcasting Next Generation Handheld (DVB-NGH) [10] proposal. Unlike 1DNUCs, 2D-NUCs cannot be created using two non-uniform Pulse Amplitude Modulation (PAM) signals. This implies that the in-phase (I) and quadrature (Q) components of the constellation symbols are no longer independent. As a consequence, a two-dimensional demapper (2D-demapper) must be applied to each received symbol. In addition, the number of Euclidean distances to be computed for Maximum Likelihood (ML) demapping of 2D-NUCs increases notably with respect to the one required for demapping 1D-NUCs [3]. This fact results into a significant increase in demapping complexity for $2 \mathrm{D}-\mathrm{NUCs}$ when compared to $1 \mathrm{D}-\mathrm{NUCs}$. However, 2D-NUCs provide larger spectral efficiency gains, approaching closely to Shannon's channel capacity limit for higher constellation orders. Therefore, ATSC 3.0 includes 2DNUCs up to 256 constellation points. Nevertheless, higher order 2D-NUCs $(1 \mathrm{~K}, 4 \mathrm{~K})$ were discarded during the standardization process due to their excessive hardware complexity.

A literature review shows abundant proposals for complexity reduction in demapping algorithms for MIMO [11] - [13] and RCs [14] - [16]. However, to the best of our knowledge, there is only one technical contribution addressing demapping techniques for 2D-NUCs [17].

This work specifically targets low complexity demappers for 2D-NUCs. A novel demapper combining two techniques based on condensation and adaptive sub-region for 2D-NUCs is proposed. It reduces latency when compared to state of art demappers [17]. Moreover, it shows a reduction in the number of Euclidean distances to be computed from $79.2 \%$ to $95.4 \%$ with respect to the ML demapper for 2D-256NUCs, 2D$1 \mathrm{kNUCs}$ and $2 \mathrm{D}-4 \mathrm{kNUCs}$. At the same time, error rate simulation results over AWGN, Rayleigh iid, DVBT-P1 and DVBT-F1 channels show comparable performance to ML demapping since the gap does not exceed $0.1 \mathrm{~dB}$ in all cases. 
This validation step includes ideal and non-ideal channel state information (CSI) conditions.

The rest of the paper is organized as follows: Section II is a literature review of low complexity demapping techniques. Section III presents the characteristics of the 2D-NUCs. Section IV shows the proposed demapper analyzing the key parameters of the demapper and the complexity reduction for each code rate. In Section V the system performance of the demapper is carried out using the BICM and OFDM platforms defined in ATSC 3.0 for ideal and non-ideal CSI. Finally, section VI gathers the main conclusions of the paper.

\section{LITERATURE REVIEW AND CONTRIBUTION}

There are different families of demapping strategies depending on the following system features: the number of antennas, the application of signal space diversity techniques (SSD) and the specific constellation shape.

In the case of MIMO systems, two approaches have been explored. The first is based on the sphere decoder [18] - [21] while the second applies lattice reduction [22] - [24]. Although these demappers provide low complexity for MIMO systems, their use in SISO systems is not necessarily appealing since the number of operations associated to the sphere decoder and lattice demapper remains large with respect to the number of constellation points in the SISO system.

Another group of proposals are related to the specific shape of the constellation being demapped. Several algorithms have been specifically designed for systems using Amplitude Phase Shift Keying (APSK) modulation. A simplified soft demapper was created for this modulation in satellite systems [25]. This demapper decomposes the APSK into two different PAM signals which, again, is not directly applicable to 2D-NUCs. In [26] a simplified demapper was proposed for the Data Over Cable Service Interface Specification (DOCSIS) 3.1 [27] where non-square quadrature amplitude modulation schemes are used. In this case independence between several bits of the symbol is exploited to simplify the demapping process. Unfortunately, this approach is not applicable to 2D-NUCs where all the bits are dependent.

On a different research thread, the inclusion of the RC technique in DVB-T2 and DVB-NGH led to several studies of low complexity demappers. In [28], authors reformulate the received rotated QAM as two PAM signals applying MMSE decorrelation. This technique is not applicable to 2D-NUCs because it is not possible to decorrelate I/Q components without high performance losses. Another family of demapping approaches reduces the area where Euclidean distances are evaluated. The latest proposal in this direction is a fixed sub-region demapper proposed in [29], [30]. The result is a reduction in complexity ranging from $25 \%$ to $50 \%$ with variable performance losses. Another interesting result can be found in [17] where a sub-region demapper applicable to 2DNUCs was proposed, following the foundations set by the previous results in [30]. This demapper provides low demapping complexity and low system performance losses when compared to the ML demapper for high code rates.
The weak aspect of this approach comes from the invariable nature of the region size since it is kept constant for all the transmission/reception scenarios. Indeed, the dimension of the region is defined empirically and it will be a fixed feature of the modulation and the coding (MODCOD) choice at the design stage. The size of the area is calculated statistically from the system performance results around the vicinity of the Low Density Parity Check (LDPC) code waterfall region. In terms of network planning, this corresponds to tweaking the broadcast system depending on LDPC code threshold for the worst supported transmission channel scenario. Concretely, the available SNR for most users will be significantly higher than the threshold SNR. Therefore due to high SNR users, the sub-region defined in [17] will be often too wide in terms of possible computation volume reduction.

An additional limitation is associated to the propagation channel variations: the sub-region size optimization was carried out for a static situation and does not adapt to the channel type. This fact will result into system performance losses for some channel scenarios. Therefore, we propose an adaptive sub-region demapper for 2D-NUCs with lower complexity and better system performance than constant subregion demappers. The dimension of the region varies for each received symbol through adaptation to the characteristics of the MODCOD, perceived Signal to Noise ratio (SNR) and channel conditions.

Sub-region demappers do not provide any reduction in latency for low code rates, where they provide negligible complexity reduction. Authors in [17] and [31] propose to complement sub-region demapping with symbol condensation techniques to further reduce latency in these corresponding regions. Inspired by this proposal, we define a metric to appropriately condensate $2 \mathrm{D}$-NUCs providing low demapping complexity with negligible performance losses. We propose to go one step further and limit the subregion algorithm to high SNR values, apply subregion plus condensation in the mid SNR range and keep simple condensation demapping on the low SNR range. Consequently, the demapper switches between these techniques depending on the corresponding system parameters [32], [33].

\section{Characteristics OF 2D-NUCS}

2D-NUCs are characterized by the complete freedom of movement of the constellation points during the design procedure. The number of degrees of freedom (DOF) when designing a M-ary 2D-NUC, where $M$ is the constellation order, is $2 M$. Already designed 2D-NUCs [3] present quadrant symmetry reducing the number of DOFs in the optimization process to $\frac{M}{2}$. This full flexibility in the design process implies higher complexity at the demapping stage. The I and Q components are mutually dependent and a 1D-demapper is no longer valid. 2D-NUCs make use of the 2D-demapper, which presents higher complexity than the 1D-demapper in terms of the number of Euclidean distances to be computed. While ML-based 1D-demapper computes $\sqrt{M}$ Euclidean distances, the ML-based 2D-demapper computes $M$ Euclidean distances. 

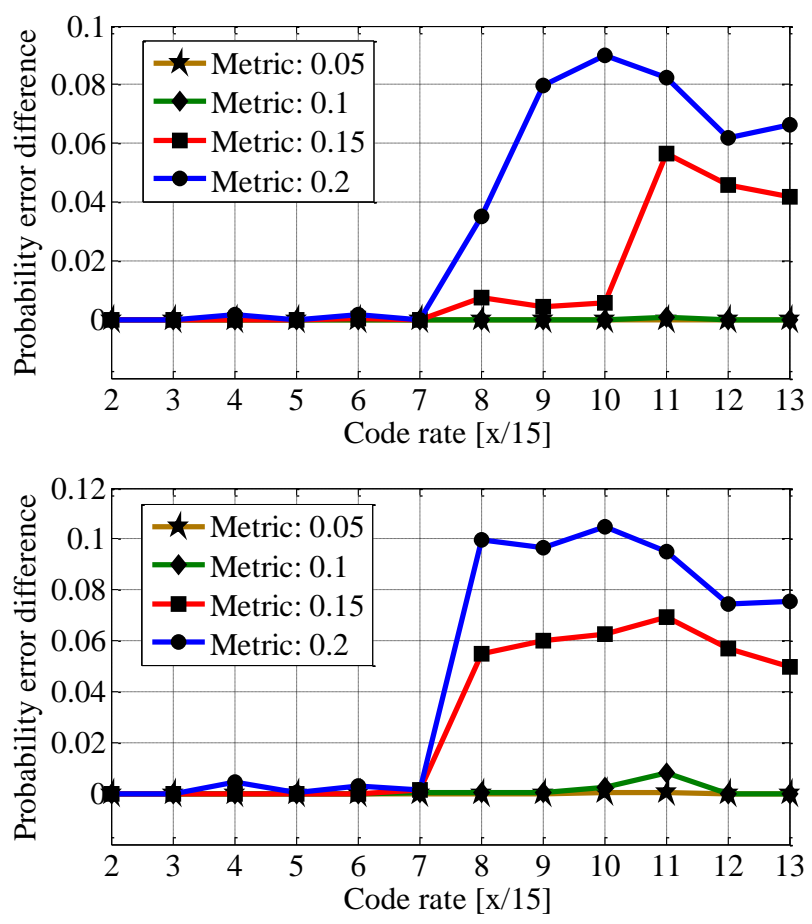

Fig. 1. Probability error difference between the ML and condensed demapper for ATSC 3.0 code rates for the AWGN channel (above) and Rayleigh iid channel (below)

The shaping of 2D-NUCs depends basically on the SNR value and channel model as stated in [3]. The design factor with the largest impact of 2D-NUCs is the SNR value. The influence of the channel model slightly shifts the positions of the constellation points without modifying the overall shape of the constellation. The SNR value is directly related to the code rate. Low SNR values correspond to low code rates and vice versa. 2D-NUCs designed for low code rates present several constellation symbols situated at the same position in the constellation diagram. This sort of constellations is called condensed 2D-NUCs [34], [35]. Condensation maximizes BICM channel capacity by favoring the capacity of the most significant bits (MSB) while sacrificing the capacity associated to the least significant bits (LSB), which in any case will be degraded by high noise values. Condensation with imperceptible capacity loss in 2D-NUCs is feasible in the low and mid code rate region.

2D-NUCs designed for the mid code rate region present circular shaping due to the maximization of the BICM channel capacity and to the application of quadrant symmetry. For high code rates, the constellation points are uniformly distributed along the constellation diagram. The shaping of 2D-NUCs for high code rates is similar to the uniform QAM.

\section{PROPOSED DEMAPPER}

In this section we define a condensation metric and present a study of the most appropriate value to provide low demapping complexity with negligible impact on performance for low code rates. For mid and high code rates, we propose an adaptive sub-region demapper. The combination of the two demapping techniques called Switched Condensed and

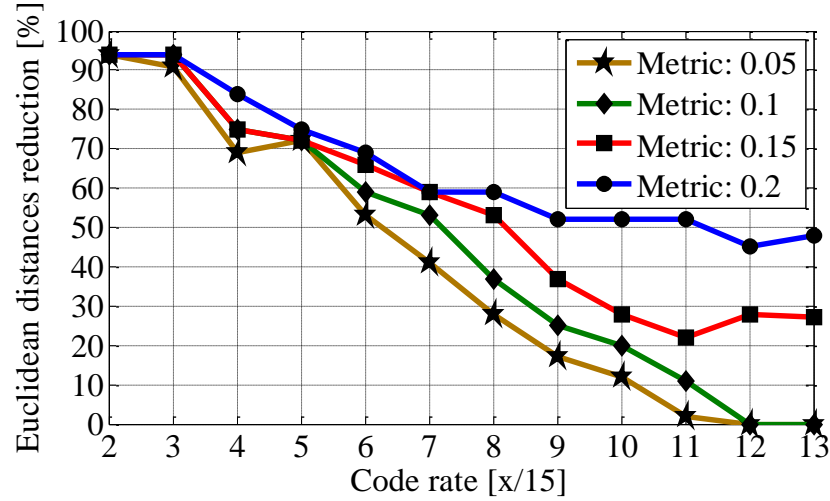

Fig. 2. Percentage of the Euclidean distances reduction between the ML and condensed demapper for ATSC 3.0 code rates

Adaptive Sub-Region (SCASR) demapper is proposed providing lower demapping complexity than state of art 2DNUC demappers with negligible impact on performance for all the code rate range. All the results shown in this section have been obtained using a fully compliant ATSC 3.0 BICM transceiver with 300 FEC blocks of length 64,800 bits [36]. Low density parity check (LDPC) code options $(R=2 / 15$ to $R=13 / 15$ ) included in ATSC 3.0 [37] are used.

\section{A. Condensed Demapper Optimization}

The condensed demapper takes advantage of the condensation applied to the 2D-NUCs designed for low and mid code rates [17], [34]. When designing a condensed demapper, the most important parameter is the condensation degree (or number of merged points) to be applied to the 2DNUCs. Most of the 2D-NUCs included in ATSC 3.0 do not present complete condensation. Indeed, points are not merged, but close to each other.

The Euclidean distance between adjacent points is used as metric for condensation. Fig. 1 shows the bit error probability difference between the ML and the condensed demapper for different metric values $(0.05,0.1,0.15$ and 0.2$)$. The bit error probability is calculated for the SNR threshold at LDPC waterfall region. ATSC 3.0 2D-256NUCs are analyzed for all code rates over AWGN and Rayleigh iid channels using the ATSC 3.0 BICM chain.

The condensed demapper presents similar bit error probability to the ML demapper for the four metric values and low code rates up to $R=7 / 15$. In this region, $2 \mathrm{D}-256 \mathrm{NUCs}$ present the highest condensation degree. From $R=8 / 15$ to $R=13 / 15$ code rates the bit error probability for 0.15 and 0.2 metrics increases significantly. Condensation applied to 2D256 NUCs within this code rate range behaves poorly. The bit error probability penalty is even higher in the case of the Rayleigh iid channel. For both channels, the metrics 0.05 and 0.1 present similar error probability to the ML demapper (except for 0.1 at $R=11 / 15$ code rate for Rayleigh iid channel).

Fig. 2 shows the reduction in terms of percentage of the number of Euclidean distances to be computed in the condensed demapper with respect to the ML demapper for all code rates. The complexity reduction increases with the value of the condensation metric. However, as shown in Fig. 1, the system performance gets worse as the metric increases. We 

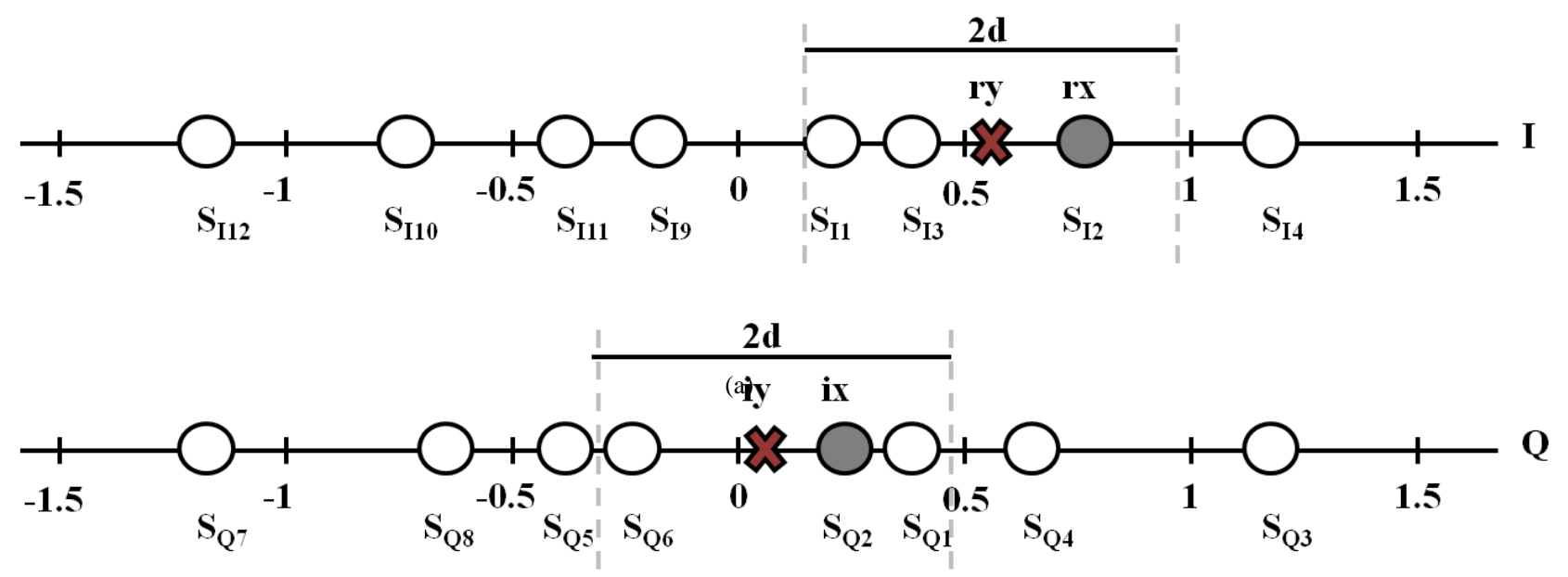

(a)

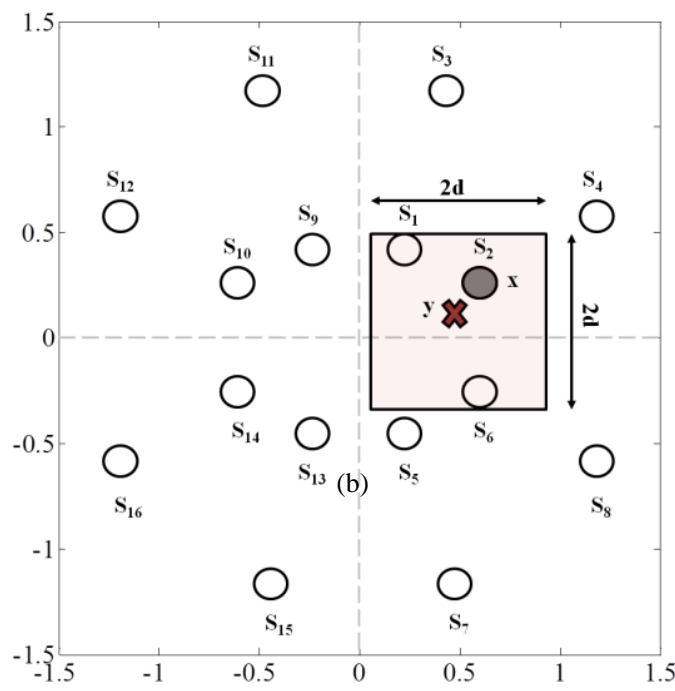

(b)

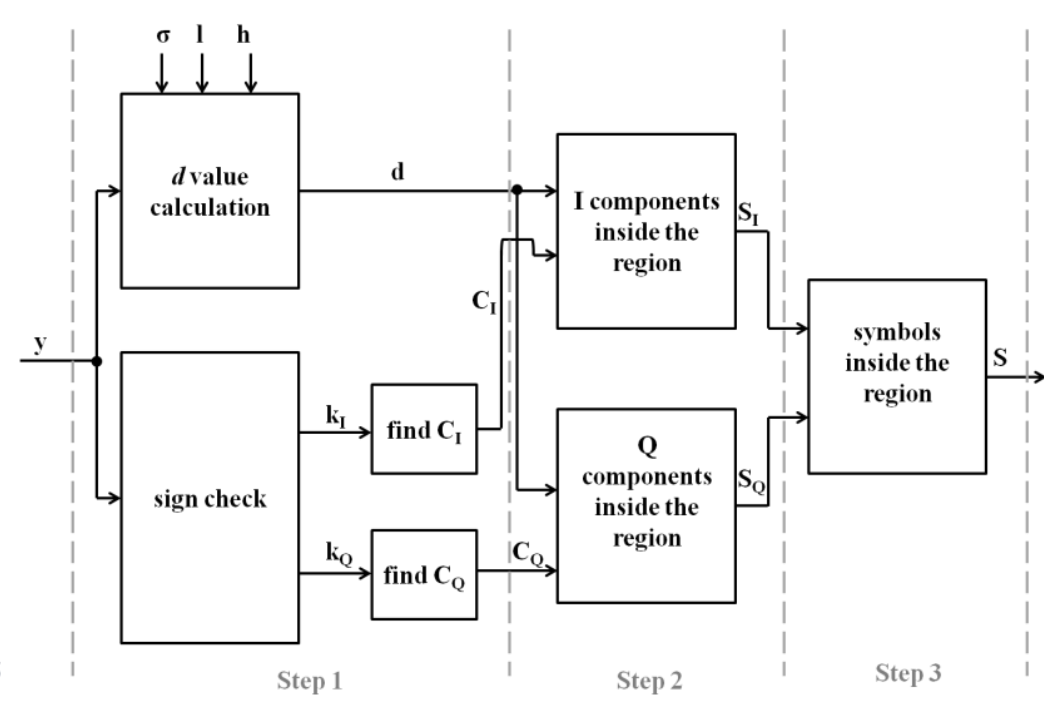

(c)

Fig. 3. a) In-phase and quadrature representation of the adaptive sub-region demapper for $2 \mathrm{D}-16 \mathrm{NUC}$ at $8 \mathrm{~dB}$. b) Constellation diagram representation of the adaptive sub-region demapper for $2 \mathrm{D}-16 \mathrm{NUC}$ at $8 \mathrm{~dB}$. c) Functional diagram of the adaptive sub-region demapper

propose to use a metric value of 0.05 as the best tradeoff between demapping complexity and system performance.

\section{B. Adaptive sub-region demapper}

The second demapper submodule makes use of the design methodology of 2D-NUCs [3]. As shown in Section III, 2DNUCs are obtained as a function of the operating SNR and the considered channel model. An adaptive sub-region demapper with a variable region size depending on these two parameters is proposed. The size varies for each received constellation symbol.

\section{1) Concept and functional diagram of adaptive sub-region demapper}

The adaptive sub-region demapper is based on computing the Euclidean distances between the received symbol and the symbols inside a square sub-region of the constellation diagram with a determined length. In the case of 2D-NUCs, we propose a square sub-region varying with the operating SNR and considered channel model. In this way, the length of the sub-region closely fits the design conditions of each 2DNUC, providing an efficient demapping process.

Fig. 3c depicts the functional diagram of the adaptive subregion demapper. The first demapping step consists of calculating the length $d$ of the sub-region (Section IV B.2) for each received symbol. In parallel, in order to calculate the closest constellation symbol to the received symbol, two processes are considered. First, a sign check of each component of the received symbol will determine the quadrant choice. The sign check is stored in $K_{I}$ (in-phase) and $K_{Q}$ (quadrature). Then, the $\mathrm{I}$ and $\mathrm{Q}$ components of the closest constellation symbol to the received symbol are obtained $\left(C_{I}\right.$ and $C_{Q}$ ) following the procedure described in Section IV B.4.

In the second step, using the $\mathrm{I}$ and $\mathrm{Q}$ components of the closest symbols $\left(C_{I}\right.$ and $\left.C_{Q}\right)$, the I and $\mathrm{Q}$ components of the 
symbols inside the sub-region are obtained and stored in arrays $\mathbf{S}_{\mathbf{I}}$ and $\mathbf{S}_{\mathbf{Q}}$, respectively (Section IV B.4).

The final step consists of merging the I and Q components of the symbols inside the sub-region, obtaining the constellation symbol set to calculate LLR values. These symbols are stored in the array $\mathbf{S}$.

A graphical description behind the idea of the adaptive subregion demapper is shown in Fig. 3a and Fig. 3b. For simplicity, the optimal 2D-16NUC for $8 \mathrm{~dB}$ of SNR is considered. This constellation is composed of 16 symbols $S_{i}$ where $i=1 \ldots 16$. Each symbol has one I and Q component associated $S_{I i}$ and $S_{Q i}$. Fig. 3a shows a graphical representation of the sub-region demapper for components I and Q. For the I component, the sub-region is limited by the I components of the constellation symbols inside the sub-region of length $2 d$ centered at $r y$, where $r y$ is the real part of the received symbol. In this case, these components are $S_{I I}, S_{I 2}$ and $S_{I 3}$. The real part of the transmitted symbol is represented as $r x$. The same applies for the Q components, being $S_{Q 1}, S_{Q^{2}}$ and $S_{Q 6}$ the Q components of the symbols inside the sub-region. Fig. $3 b$ shows the sub-region in the constellation diagram and the symbols inside it. The transmitted symbol is represented as $x$ and the received symbol as $y$. Symbols $S_{1}, S_{2}$ and $S_{6}$ are inside the sub-region for the particular $x$ and $y$ symbols.

\section{2) Length of the square adaptive sub-region demapper}

The value of the length of the sub-region is based on having, at least, the transmitted symbol inside the sub-region. Constellation symbols situated at the limit of the sub-region are also included. Equation (1) shows the calculation of the received symbol in a communication system with Gaussian noise for a determined channel.

$$
y=h x+n
$$

Where $y$ is the received symbol, $x$ the transmitted symbol, $n$ the noise value (in this case Gaussian noise is assumed) and $h$ the fading coefficients of the channel. All the parameters are complex values. Manipulating (1), the position of the transmitted symbol $x$ is obtained as:

$$
x=\frac{y}{h}-\frac{n}{h}
$$

The first term $\frac{y}{h}$ refers to a zero forcing equalization. Manipulating the second term in (2), we derive the length of the sub-region $d$ in (3). This length is calculated for each received symbol, i.e., it adapts to the channel and noise variations.

$$
d=\left|\frac{(l+j l) \sigma}{h}\right|
$$

Where $\sigma$ is the standard deviation of the Gaussian distribution and $l$ is the maximum random value which determines the percentage of values that lie within a band around the mean in a normal distribution. As shown in (3), the value of the length is directly proportional to the noise value. The higher the noise value the higher the length value and vice versa.

\section{3) Design of adaptive sub-region demapper}

The adaptive sub-region demapper presents a series of special cases. The first case is considered when there is no symbol inside the sub-region. In this case, the length of the sub-region is increased until at least, two symbols are inside. Second, in the calculation of the log-likelihood ratio (LLR) values for each transmitted bit, the conditional probability of receiving $y$ given that the $b_{i}$ transmitted is $0 p\left(y \mid b_{i}\right.$ is 0$)$ and the conditional probability of receiving $y$ given that $b_{i}$ transmitted is $1 p\left(y \mid b_{i}\right.$ is 1) are needed. However, it can occur that for a determined $b_{i}$ all the symbol values inside the sub-region are 0 or 1 for that particular bit. In this case, taking into account that a FEC soft decoder performs better under pessimistic assumptions (consider one symbol as if it was closer than its real position) the conditional probability of the missing bit value is calculated for the furthest symbol inside the subregion. This can derive into both conditional probabilities with the same value if the Euclidean distances are the same for both bit values. This is solved by increasing the length value and forcing that there are, at least, two symbols with different Euclidean distances.

This solution performs efficiently for low and mid code rates (up to $R=9 / 15$ ). However, for higher code rates, system performance gets worse. For low code rates, the sub-region covers mainly the totality of constellation points and this demapper does not reduce complexity. For high code rates the sub-region only covers part of the constellation diagram and the case explained above in which one bit value is not inside the sub-region commonly happens for the two MSBs. In this case, another approach is proposed: compute the Euclidean distance from the received point $y$ to the origin of the constellation diagram $(0+0 \mathrm{j})$ since it represents the intersection of the four quadrants. This option requires only one Euclidean distance to be computed. As shown in Section V, this solution has negligible impact on the demapping complexity and system performance.

Fig. 4 shows the percentage reduction in the number of Euclidean distances to be computed w.r.t. ML demapper. For low code rates, limited reduction is observed. In this zone, where the noise components are high, the length of the subregion is highly increased gathering almost all the constellation points. In the region of mid code rates, as the noise value is lower, the number of points inside the subregion is lower and the number of Euclidean distances to be computed is reduced. High code rates present the highest complexity reduction as the length of the sub-region is highly reduced.

The adaptive sub-region demapper proposed in this section provides high demapping complexity reduction in the region of mid and high code rates with negligible performance loss. A complexity reduction from $60 \%$ for $R=7 / 15$ to $95 \%$ for $R=13 / 15$ is achieved. In order to decrease the complexity for low and mid code rates, a combination of the adaptive subregion and condensed demapper is proposed (Section IV C).

\section{4) Determination of points inside the adaptive sub-region}

The efficient determination of the points inside the subregion is a key aspect. We propose a methodology based on finding the closest constellation symbol to the received symbol. Then, a comparison process starting from this symbol is performed in order to determine the first point outside the sub-region. 


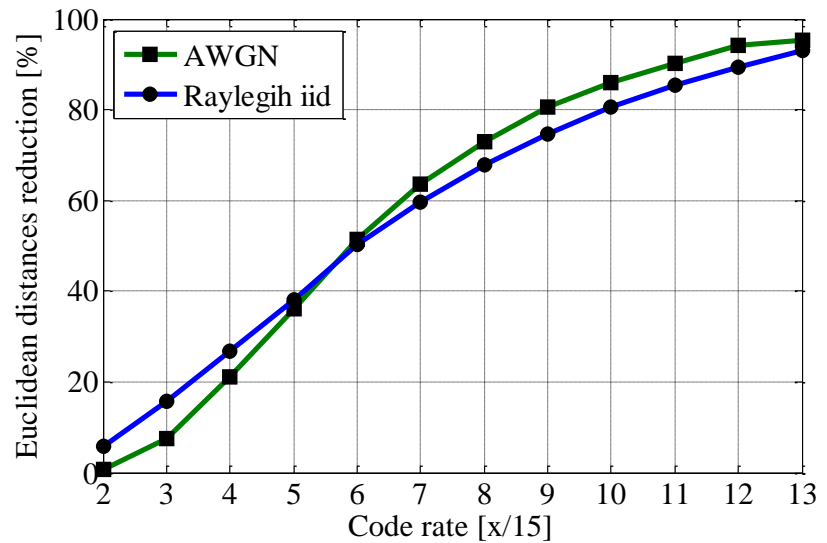

Fig. 4. Percentage of the Euclidean distances reduction between the ML and adaptive sub-region demapper for ATSC 3.0 code rates for the AWGN and Rayleigh iid channels.

The procedure is explained for one axis (I) and the methodology is then repeated for the other axis (Q). First, a sign check of the I component of the received symbol is performed in order to determine the quadrant. Next, dichotomy process is applied to the real part of the constellation points in order to determine the closest constellation point to the received point. The dichotomy process consists of dividing the set of I components into two subsets with equal number of components. The I component of the received symbol is compared with the limit value of the two subsets. A subset is chosen based on this comparison. An iterative procedure is performed until there is only one I component in the subset. This I component belongs to the closest symbol. Next, starting from the I component of the closest constellation point, comparisons are performed between the real part of the constellation symbols and the value of the length of the sub-region. In this way, the number of comparisons performed is reduced, i.e., the number of the points inside the sub-region plus the first one outside the region having a larger value than the considered length.

\section{5) Determination of l value}

The next step consists of determining the most suitable value of $l$. We approximate (3) to a normal distribution to set $l$ $=3$ as initial value. In this way, we assume that the transmitted symbol will be inside the sub-region in a high percentage of time. Then, an iterative procedure is performed to obtain the optimal value of $l$. Table I shows the study carried out to determine the optimal value of $l$ with the lowest demapping complexity jointly with system performance losses below 0.1 $\mathrm{dB}$. Steps of 0.1 are considered for the determination of the $l$ value. Results from Table I show that for $1=3.6$, the performance penalty of the adaptive sub-region demapper w.r.t. the ML demapper is below $0.1 \mathrm{~dB}$ for all code rates. For $R=2 / 15$ and $R=6 / 15$ a value of $1=3$ also provides a negligible performance penalty and the same applies for $R=10 / 15$ code rate with $1=3.3$. These values imply lower demapping complexity than for $1=3.6$ (in the range of $2-5 \%$ lower demapping complexity). For simplicity and targeting a hardware implementation, we take $l=3.6$.

It is important to note that the value $l=3.6$ is also valid for the ATSC 3.0 OFDM platform under ideal CSI. However, for non-ideal CSI the same study has been done obtaining a new value of $l=5$.

\section{Switched condensed and adaptive sub-region demapper}

Being a combination of the two proposed demapping techniques, the combined SCASR demapper applies the metric value of 0.05 for the underlying condensed demapper and $l=$ 3.6 for the adaptive sub-region demapper. For these parameters, observed performance remains within $0.1 \mathrm{~dB}$ from the ML demapper. Fig. 5 shows the functional diagram of the SCASR demapper. The first block corresponds to the decision block where, depending on the code rate, either one or both demappers are switched on. The criteria depend on the code rate range: for the code rates of $R=2 / 15$ and $R=3 / 15$ only the condensed demapper is applied. From code rates $R=4 / 15$ to $R=10 / 15$, both demappers (condensed and adaptive subregion) are used. From code rates of $R=11 / 15$ to $R=13 / 15$, only the adaptive sub-region demapper is used.

Fig. 6 shows the reduction in terms of percentage of the number of Euclidean distances to be computed w.r.t. ML demapper. The SCASR demapper presents a demapping complexity reduction ranging from $77 \%$ to $95 \%$ for the AWGN channel. This reduction ranges between $79 \%$ and $93 \%$ in the case of the Rayleigh iid channel. For this latter channel type, the fading coefficients and the SNR value (higher than in the AWGN channel for error-free decoding) increase the length of the sub-region.

The influence of the condensed demapper is remarkable for low code rates as it provides low demapping complexity. The adaptive sub-region demapper performs at its best for high code rates. In the range of mid code rates, both demappers have similar influence in the complexity reduction.

\section{Hardware implications}

The additional hardware complexity of the SCASR demapper w.r.t. the ML or Max-Log demappers is the calculation of the length of the sub-region and the constellation points inside the sub-region. Regarding the calculation of the parameter $d$, the remarkable operations in terms of hardware requirements are one multiplication and one division. The multiplication can be easily implemented via an addition since the SNR is constant for all the received symbols. The division can be considered equivalent to an Euclidean distance computation and it is taken into account in the complexity analysis in Section V. The rest of operations are comparisons or additions which are negligible if compared with Euclidean distance computation.

\section{PERformance Evaluation}

\section{A. Demapping complexity and system performance}

In this section, the system performance of the proposed SCASR demapper is analyzed. As a contribution, in this paper the results of a low complexity demapper for $2 \mathrm{D}-256 \mathrm{NUCs}$ are presented using, for the first time, a fully compliant ATSC 3.0 OFDM transmitter - receiver chain. The transmitted signal is composed of 300 FEC blocks with 64,800 bit length. Four different channel models are simulated: AWGN, Rayleigh iid for the BICM system and DVB-T P1 (Rayleigh) and DVB-T F1 (Rice) [38] for the OFDM system. Four different code rates 
TABLE I

SEARCH OF THE OPTIMAL L VALUE

\begin{tabular}{|c|c|c|c|c|c|c|c|c|}
\hline \multirow{3}{*}{ Channel } & \multirow{3}{*}{$\begin{array}{l}\text { Code rate } \\
\text { [x/15] }\end{array}$} & \multicolumn{7}{|c|}{1 value } \\
\hline & & 3 & 3.1 & 3.2 & 3.3 & 3.4 & 3.5 & 3.6 \\
\hline & & \multicolumn{7}{|c|}{ System performance losses $(\mathrm{dB})$ of the adaptive sub-region w.r.t. the ML demapper } \\
\hline \multirow{4}{*}{ AWGN } & $R=2$ & $<0.1$ & $<0.1$ & $<0.1$ & $<0.1$ & $<0.1$ & $<0.1$ & $<0.1$ \\
\hline & $R=6$ & $<0.1$ & $<0.1$ & $\angle 0.1$ & $\angle 0.1$ & $\angle 0.1$ & $\angle 0.1$ & $<0.1$ \\
\hline & $R=10$ & $<0.1$ & $<0.1$ & $<0.1$ & $<0.1$ & $<0.1$ & $<0.1$ & $<0.1$ \\
\hline & $R=13$ & 3.3 & 3.3 & 1.9 & 1.7 & 0.5 & 0.3 & $<0.1$ \\
\hline \multirow{4}{*}{ Rayleigh iid } & $R=2$ & $<0.1$ & $<0.1$ & $<0.1$ & $<0.1$ & $<0.1$ & $<0.1$ & $<0.1$ \\
\hline & $R=6$ & $<0.1$ & $<0.1$ & $<0.1$ & $<0.1$ & $<0.1$ & $<0.1$ & $<0.1$ \\
\hline & $R=10$ & 1.1 & 1.1 & 1.1 & $<0.1$ & $<0.1$ & $<0.1$ & $<0.1$ \\
\hline & $R=13$ & 1.5 & 0.8 & 0.5 & 0.3 & 0.3 & 0.3 & $<0.1$ \\
\hline
\end{tabular}

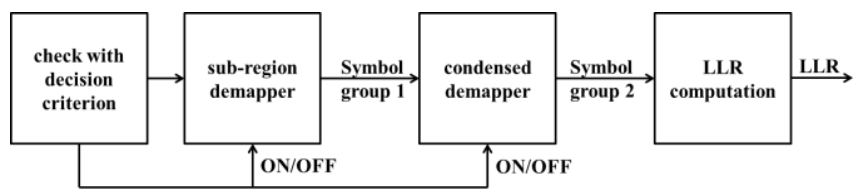

Fig. 5. Functional diagram of the SCASR demapper

are used $(R=2 / 15, R=6 / 15, R=10 / 15$ and $R=13 / 15)$. In the OFDM system, all the interleavers defined in ATSC 3.0 for single Physical Layer Pipe (PLP) are used. Table II shows the results for ideal CSI. No pilots are used and perfect synchronization is considered.

Complexity results consider the mean of all of the computed Euclidean distances along the simulation duration.

In all channel conditions, results from Table II show that the SCASR performance is comparable to the ML demapper with a negligible gap (under $0.1 \mathrm{~dB}$ ). Meanwhile, complexity reduction from $6 \%$ to $27 \%$ w.r.t. the state of art demappers for 2D-NUCs [17] and from $79.2 \%$ to $95.4 \%$ w.r.t. the ML demapper is obtained.

Table III extends the results for non-ideal CSI. The value of 1 is 5 as explained in Section IV B5. All the interleavers defined in ATSC 3.0 for single PLP are used, the pilot pattern is SP12_2, 16K FFT is used and the guard interval is 1024 samples. The pilot boosting is $4.7 \mathrm{~dB}$. Two-dimensional channel estimation based on the Discrete Fourier Transform (DFT) method [39] is performed jointly with frequency and time filtering.

Results in Table III validate the proposed SCASR demapper under non-ideal CSI. The percentages of the demapping complexity reduction are quite similar to the ideal CSI case (from $8.7 \%$ to $23 \%$ w.r.t. [17] and from $71.8 \%$ to $93.7 \%$ w.r.t. the ML demapper). The corresponding performance penalties on DVB-T P1 channel conditions are 0.1 and $0.2 \mathrm{~dB}$ for $R=10 / 15$ and $R=13 / 15$ code rates respectively.

\section{B. Extension to $2 D-1 k N U C$ and $2 D-4 k N U C$}

The main problem for high order 2D-NUCs ( $1 \mathrm{k}$ and $4 \mathrm{k}$ ), is their design and demapping complexity. Indeed, proposed

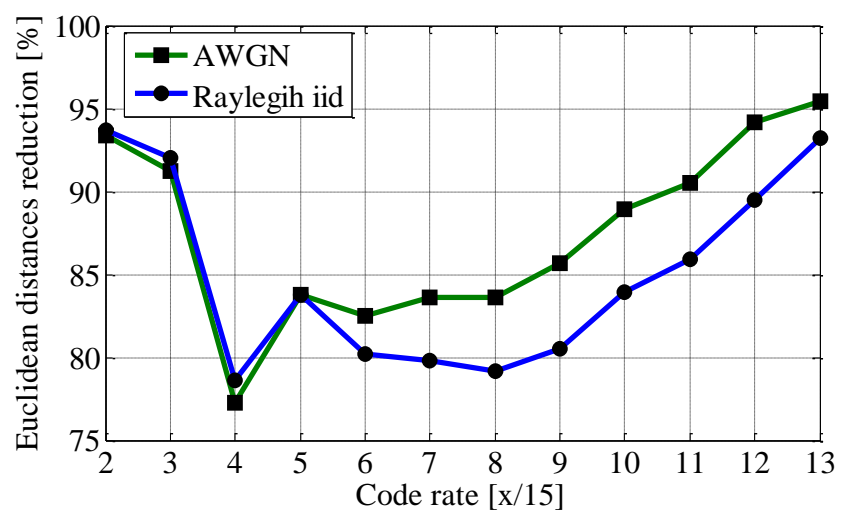

Fig. 6. Percentage of the Euclidean distances reduction between the ML and condensphere demapper for ATSC 3.0 code rates for the AWGN and Rayleigh iid channels.

NUCs in ATSC 3.0 for these constellation orders are 1DNUCs. As a contribution, the system performance and complexity reduction of the SCASR demapper for such high order constellations is presented. In a previous work, the authors proposed in [34] 2D-1kNUCs and 2D-4kNUCs. 2D$1 \mathrm{kNUC}$ for $R=5 / 15$ code rate and $2 \mathrm{D}-4 \mathrm{kNUC}$ for $R=3 / 15$ code rate are used in this study. Ideal CSI is considered.

Results in Table IV validate the use of the SCASR demapper for high order 2D-NUCs. Demapping complexity reduction ranges from $96.5 \%$ to $99.7 \%$ (AWGN) and from $96 \%$ to $99.1 \%$ (Rayleigh iid). In all cases, performance penalty remains within $0.1 \mathrm{~dB}$ from the ML demapper. High order 2D-NUCs could be taken into account in future communications systems with the use of low complexity demappers such as the SCASR demapper.

\section{CONCLUSIONS}

In this paper, a novel low complexity demapper switching between two different demapping techniques based on condensation and on adaptive sub-region (SCASR) is proposed for 2D-NUCs. First, the main characteristics of the 2D-NUCs are identified in order to design a demapper which adapts to these characteristics. Clear criteria are proposed for 
TABLE II

SYSTEM PERFORMANCE AND DEMAPPING COMPLEXITY REDUCTION OF THE SCASR DEMAPPER FOR IDEAL CSI

\begin{tabular}{|c|c|c|c|c|c|}
\hline \multirow{2}{*}{ Channel } & \multirow{2}{*}{ Parameter } & \multicolumn{4}{|c|}{ Code rate $[\mathrm{x} / 15]$} \\
\hline & & $R=2$ & $R=6$ & $R=10$ & $R=13$ \\
\hline \multirow{3}{*}{ AWGN } & ML: SNR [dB] & 1.7 & 10.6 & 17.2 & 22.3 \\
\hline & $\begin{array}{c}\text { SCASR Losses } \\
{[\mathrm{dB}]} \\
\end{array}$ & $<0.1$ & $<0.1$ & $<0.1$ & $<0.1$ \\
\hline & $\begin{array}{c}\text { Complexity } \\
\text { Reduction [\%] }\end{array}$ & 93.4 & 82.5 & 88.9 & 95.4 \\
\hline \multirow{3}{*}{$\begin{array}{l}\text { Rayleigh } \\
\text { iid }\end{array}$} & $\begin{array}{l}\text { ML: SNR } \\
\end{array}$ & 2.9 & 12.9 & 20.1 & 26.7 \\
\hline & $\begin{array}{c}\text { SCASR Losses } \\
{[\mathrm{dB}]} \\
\end{array}$ & $<0.1$ & $<0.1$ & $<0.1$ & $<0.1$ \\
\hline & $\begin{array}{c}\text { Complexity } \\
\text { Reduction [\%] }\end{array}$ & 93.7 & 80.2 & 83.9 & 93.2 \\
\hline \multirow{3}{*}{$\mathrm{P} 1$} & ML: SNR & 2.3 & 12.5 & 19.7 & 26.6 \\
\hline & $\begin{array}{c}\text { SCASR Losses } \\
{[\mathrm{dB}]} \\
\end{array}$ & $<0.1$ & $<0.1$ & $<0.1$ & $<0.1$ \\
\hline & $\begin{array}{c}\text { Complexity } \\
\text { Reduction [\%] }\end{array}$ & 93.7 & 79.2 & 81.5 & 92.2 \\
\hline \multirow{3}{*}{$\mathrm{F} 1$} & $\begin{array}{l}\text { ML: SNR } \\
\end{array}$ & 1.3 & 10.5 & 17.1 & 22.3 \\
\hline & $\begin{array}{c}\text { SCASR Losses } \\
{[\mathrm{dB}]} \\
\end{array}$ & $<0.1$ & $<0.1$ & $<0.1$ & $<0.1$ \\
\hline & $\begin{array}{c}\text { Complexity } \\
\text { Reduction [\%] }\end{array}$ & 93.7 & 80.1 & 86.7 & 94.6 \\
\hline
\end{tabular}

the switching between the two underlying demappers providing the best performance. The functional diagram of the SCASR demapper is shown and the configuration of the submodules is detailed for the code rates defined in ATSC 3.0.

The first contribution of the work is the design of an adaptive sub-region demapper closely suited to the shaping of 2D-NUCs. The dimension of the sub-region with best compromise between performance and complexity is proposed. The design and functional diagram of the demapper are also defined. This demapper presents high demapping complexity reduction for high and mid code rates.

For low code rates, the use of a condensed demapper is proposed. A metric to appropriately condense 2D-NUCs to provide low demapping complexity with negligible impact on performance represents the second contribution.

In order to validate the proposed SCASR demapper, simulation results are presented using a fully compliant ATSC 3.0 OFDM transceiver. Obtained performance remains within $0.1 \mathrm{~dB}$ from the ML demapper. Meanwhile, a significant reduction in the number of computed Euclidean distances ranging from $79.2 \%$ to $95.4 \%$ is obtained when compared to the ML demapper. When other 2D-NUC demapping proposals are considered, the SCASR demapper still shows lower complexity (from $6 \%$ to $27 \%$ ) and performs better (degradation always below $0.1 \mathrm{~dB}$ ).

For non-ideal CSI, similar performance results and complexity reductions as for ideal CSI are still obtained. An extension of this demapper is proposed for high order 2DNUCs showing a complexity reduction of up to $99.7 \%$ with respect to $\mathrm{ML}$ demapping.
TABLE III

SYSTEM PERFORMANCE AND DEMAPPING COMPLEXITY REDUCTION OF THE SCASR DEMAPPER FOR NON-IDEAL CSI

\begin{tabular}{|c|c|c|c|c|c|}
\hline \multirow{2}{*}{ Channel } & \multirow{2}{*}{ Parameter } & \multicolumn{4}{|c|}{ Code rate $[\mathrm{x} / 15]$} \\
\hline & & $R=2$ & $R=6$ & $R=10$ & $R=13$ \\
\hline \multirow{3}{*}{$\mathrm{P} 1$} & ML: SNR [dB] & 3.4 & 13.3 & 20.3 & 27.7 \\
\hline & $\begin{array}{c}\text { SCASR Losses } \\
{[\mathrm{dB}]}\end{array}$ & $<0.1$ & $<0.1$ & 0.1 & 0.2 \\
\hline & $\begin{array}{c}\text { Complexity } \\
\text { Reduction [\%] }\end{array}$ & 93.7 & 74.2 & 75.2 & 90 \\
\hline \multirow{3}{*}{$\mathrm{F} 1$} & $\begin{array}{c}\text { ML: SNR } \\
\end{array}$ & 2.2 & 11.1 & 17.5 & 22.8 \\
\hline & $\begin{array}{c}\text { SCASR Losses } \\
{[\mathrm{dB}]}\end{array}$ & $<0.1$ & $<0.1$ & $<0.1$ & $<0.1$ \\
\hline & $\begin{array}{c}\text { Complexity } \\
\text { Reduction [\%] }\end{array}$ & 93.7 & 71.8 & 78.7 & 91.1 \\
\hline
\end{tabular}

TABLE IV

SYSTEM PERFORMANCE AND DEMAPPING COMPLEXITY REDUCTION OF SCASR DEMAPPER FOR HIGH ORDER 2D-NUC

\begin{tabular}{||c||c||c|c||}
\hline \multirow{4}{*}{ Channel } & \multicolumn{1}{|c||}{ Parameter } & \multicolumn{2}{c|}{$\begin{array}{c}\text { Constellation order \& code } \\
\text { rate }\end{array}$} \\
\cline { 3 - 4 } & & $\begin{array}{c}\text { 2D-1kNUC } \\
\& R=5 / 15\end{array}$ & $\begin{array}{c}\text { 2D-4kNUC } \\
\& R=3 / 15\end{array}$ \\
\hline \hline \multirow{2}{*}{ AWGN } & $\begin{array}{c}\text { SCASR } \\
\text { Losses [dB] }\end{array}$ & $<0.1$ & $<0.1$ \\
\cline { 2 - 4 } & $\begin{array}{c}\text { Complexity } \\
\text { Reduction [\%] }\end{array}$ & 96.5 & 99.7 \\
\hline \hline \multirow{3}{*}{$\begin{array}{c}\text { Rayleigh } \\
\text { iid }\end{array}$} & $\begin{array}{c}\text { SCASR } \\
\text { Losses [dB] }\end{array}$ & $<0.1$ & $<0.1$ \\
\cline { 2 - 4 } & $\begin{array}{c}\text { Complexity } \\
\text { Reduction [\%] }\end{array}$ & 96 & 99.1 \\
\hline
\end{tabular}

Thanks to this proposal, the design of broadcasting systems which closely match Shannon's channel capacity limit for high spectral efficiency ranges becomes within feasible reach.

\section{REFERENCES}

[1] A. Goldsmith, S.A. Jafar, N. Jindal, and S. Vishwanath, "Capacity limits of MIMO channel," IEEE Journal on Selected Areas in Communications, vol. 21, no. 5, pp. 684-702, June 2003.

[2] C. Abdel Nour and C. Douillard, "Rotated QAM constellation to improve BICM performance for DVB-T2," in Proc. ISSSTA, pp. 354359, Aug. 2008.

[3] J. Barrueco et al., "Constellation Design for Bit-interleaved Coded Modulation (BICM) Systems in Advanced Broadcast Standards," IEEE Trans. Broadcast., DOI: 10.1109/TBC.2017.2677259.

[4] ATSC Standard: Physical Layer Protocol, document A/322:2016, Adv. Telev. Syst. Comm., Sep. 2016.

[5] D. Gomez-Barquero, et al., "MIMO for ATSC 3.0," IEEE Trans. Broadcast., vol 62, no. 1, pp. 298-305, March 2016.

[6] N. S. Loghin et al., "Non-uniform constellations for ATSC 3.0," IEEE Trans. Broadcast., vol. 62, no. 1, pp. 197-203, Mar. 2016.

[7] J. Zoellner and N. Loghin, "Optimization of high-order nonuniform QAM constellations," in Proc. IEEE Int. Symp. Broadband Multimedia Syst. Broadcast., London, U.K., Jun. 2013, pp. 1-6.

[8] B. Mouhouche, D. Ansorregui, and A. Mourad, "High Order 
Non-Uniform Constellations for Broadcasting UHDTV," Proceedings of IEEE Wireless Communications and Networking Conference (WCNC), Istanbul, Turkey, pp. 600-605, Apr. 2014.

[9] J. Barrueco et al., "Combining advanced constellations and SSD techniques for optimal BICM capacity," in Proc. IEEE Int. Symp. Broadband Multimedia Syst. Broadcast. (BMSB), Ghent, Belgium, pp. 1-4, Jun. 2015.

[10] D. Gomez-Barquero, C. Douillard, P. Moss, and V. Mignone, "DVBNGH: The next generation of digital broadcast services to handheld devices," IEEE Trans. Broadcast., vol. 60, no. 2, pp. 246-257, Jun. 2014.

[11] T-J. Hsieh, and W-H. Sheen, "A Lattice-Reduction-Aided Max-Log List Demapper for Coded MIMO Receivers," IEEE Trans. Vehicular Technology., vol. 63, no. 2, pp. 664-673, Aug. 2013.

[12] E. C. Y. Peh and Y.-C. Liang, "Power and modulo loss tradeoff with expanded soft demapper for LDPC coded GMD-THP MIMO systems," IEEE Trans. Wireless Commun., vol. 8, no. 2, pp. 714-724, Feb. 2009.

[13] X-F. Qi, and K. Holt, "A Lattice-Reduction-Aided Soft Demapper for High-Rate Coded MIMO-OFDM Systems," IEEE Signal Processing Letters, vol. 14, no. 5, pp. 305-308, Apr. 2007.

[14] S. Tomasin, and M. Butussi, "Low Complexity Demapping of Rotated and Cyclic Q Delayed Constellations for DVB-T2," IEEE Wireless Communications Letters, vol. 1, no. 2, pp. 81-84, Jan. 2012.

[15] M. Saleh, M. Sadek, and S. El Ramly, "Novel hybrid soft-hard demodulation schemes for RQD constellation," in Proc. Int. Conf. Telecommun. Signal Process., pp. 684-688, Jul. 2012.

[16] A. R. Jafri, A. Baghdadi, M. Waqas, and M. Najam-Ul-Islam, "HighThroughput and Area-Efficient Rotated and Cyclic Q Delayed Constellations Demapper for Future Wireless Standards," IEEE Access., vol. 5, pp. 3077-3084, Jan. 2017.

[17] M. Fuentes, D. Vargas, and D. Gómez-Barquero, "Low-Complexity Demapping Algorithm for Two-Dimensional Non-Uniform Constellations," IEEE Trans. Broadcst., vol. 62, no. 2, pp. 375-383, June 2016.

[18] E. Viterbo, and J. Boutros, "A universal lattice code decoder for fading channels," IEEE Trans. Information Theory., vol. 45, no. 5, pp. 16391642, Aug. 2002.

[19] Z. Guo, and P. Nilsson, "Algorithm and implementation of the K-best sphere decoding for MIMO detection," IEEE Journal on Selected Areas in Communications, vol. 24, no. 3, pp. 491-503, March 2006.

[20] C. Studer, A. Burg, and H. Bolcskei, "Soft-output sphere decoding: algorithms and VLSI implementation," IEEE Journal on Selected Areas in Communications, vol. 26, no. 2, pp. 684-702, Feb. 2008.

[21] C. Barjau, M. Fuentes, T. Shitomi, and D. Gomez-Barquero, "MIMO Sphere Decoder with Successive Interference Cancellation for TwoDimensional Non-Uniform Constellations," IEEE Communications Letters, DOI: 10.1109/LCOMM.2017.2653775.

[22] E. Agrell, T. Eriksson, A. Vardy, and K. Zeger, "Closest point search in lattices," IEEE Trans. Information Theory, vol. 48, no. 8, pp. 22012214, Aug. 2002.

[23] M. O. Damen, H. El Gamal, and G. Caire, "On maximum-likelihood detection and the search for the closest lattice point," IEEE Trans. Information Theory, vol. 49, no. 10, pp. 2389-2402, Oct. 2003.

[24] A. Haroun, C. A. Nour, M. Arzel, and C. Jego, "Low-Complexity Soft Detection of QAM Demapper for a MIMO System," IEEE Communications Letters, vol. 20, no. 4, pp. 732-735, Feb. 2016.

[25] Q. Xie, Z. Wang, and Z. Yang, "Simplified Soft Demapper for APSK with Product Constellation Labelling," IEEE Trans. Wireless Communications, vol. 11, no. 7, pp. 2649-2657, May 2012.

[26] J. Lee, J-Y. Jung, and J. M. Ahn, "Simplified Non-Square Amplitude Modulation Demapper for DOCSIS 3.1," IEEE Trans. Broadcsating, vol. 63, no. 1, pp. 156-161, Dec. 2016.

[27] "Data-over-cable service interface specifications DOCSIS 3.1-physical layer specification," Cable Televis. Lab. (CableLabs), Louisville, CO, USA, Tech. Rep. CM-SP-PHYv3.1-I09-160602, Jun. 2016.
[28] K. Kim, N. Basutkar, K. Bae, P. Xue, and H. Yang, "One-Dimensional Soft-Demapping Algorithms for Rotated QAM and Software Implementation on DSP," IEEE Trans. Signal Processing, vol. 61, no. 15, pp. 3918-3930, May 2013.

[29] D. Pérez-Calderón, V. Baena-Lecuyer, A. C. Oria, P. López, and J. G. Doblado, "Rotated constellation demapper for DVB-T2," IEEE Electron. Lett., vol. 47, no. 1, pp. 31-32, Jan. 2011.

[30] D. Perez-Calderon, V. Baena-Lecuyer, A. C. Oria, P. Lopez, and J. G. Doblado, "Simplified rotated constellation demapper for secon generation terrestrial digital video broadcasting," IEEE Trans. Broadcast., vol. 59, no. 1, pp. 160-167, Mar. 2013.

[31] S. Kwon et al., "Simplified non-uniform constellation demapping scheme for the next broadcasting system," in Proc. IEEE Int. Symp. Broadband Multimedia Syst. Broadcast. (BMSB), Jun. 2015.

[32] Y. Tao, H-J. Kim, S. Jeon, and J-S. Seo,"Low-complexity switched soft demapper for rotated non-uniform constellation DVB-NGH systems," in Proc. IEEE Int. Symp. Broadband Multimedia Syst. Broadcast. (BMSB), Jun. 2014

[33] Y. Tao, H-J. Kim, S. Jeon, and J-S. Seo, "Low Complexity Switched Soft Demapper for Rotated QAM Constellations," IEEE Trans. Broadcast., vol. 62, no. 3, pp. 532-539, Jun. 2016.

[34] J. Barrueco et al., "Low complexity and high order two-dimensional non-uniform constellations for high capacity broadcasting systems," in Proc. IEEE Int. Symp. Broadband Multimedia Syst. Broadcast. (BMSB), Jun. 2016.

[35] J. Barrueco et al., "Condensation Methodologies for Two-Dimensional Non-Uniform Constellations," in Proc. IEEE Int. Symp. Broadband Multimedia Syst. Broadcast. (BMSB), Jun. 2017.

[36] L. Michael and D. Gomez-Barquero, "Bit-interleaved coded modulation (BICM) for ATSC 3.0," IEEE Trans. Broadcast., vol. 62, no. 1, pp. 181-188, Mar. 2016.

[37] K.-J. Kim et al., "Low-density parity-check codes for ATSC 3.0," IEEE Trans. Broadcast., vol. 62, no. 1, pp. 189-196, Mar. 2016.

[38] ETSI, "Digital Video Broadcasting (DVB); Implementation Guidelines for a Second Generation Digital Terrestrial Television Broadcasting System (DVB-T2), " document ETSI TS 102831 V1.2.1, ETSI, Sophia Antipolis, France, Aug. 2012.

[39] 0. Edfors, M. Sandell, J.-J. van de Beek, S.K. Wilson and P. 0. Borjesson, Analysis of DFT-based channel estimators for OFDM. Research Report TULEA 1996:17, Div. of Signal Processing, Luleå University of Technology, Sept. 1996. 